\title{
First report of Cucumis melo endornavirus on Cucumis melo in China
}

\author{
Rong Zeng ${ }^{1} \cdot$ Lihui $\mathrm{Xu}^{1} \cdot$ Shigang Gao ${ }^{1} \cdot$ Zhiwei Song $^{1} \cdot$ Ping Gao $^{1} \cdot$ Fuming Dai ${ }^{1}$ \\ Received: 26 February 2020 / Accepted: 30 July 2020 / Published online: 6 August 2020 \\ (C) Società Italiana di Patologia Vegetale (S.I.Pa.V.) 2020
}

Keywords CmEV $\cdot$ Endornavirus $\cdot$ Small RNA

Melon (Cucumis melo) is a very popular fruit in china, and its production has been estimated around 12,788,218 tons cultivated in more than 358,961 ha in 2018 (FAOSTAT 2020). In 2019 , virus-like symptoms such as stunting and leaf malformation were observed on melon in a high tunnel in Shanghai, China. A sample of symptomatic melon leaves was collected and total RNAs were extracted using the TRIzol method following manufacturer's instructions (Thermo Fisher Scientific, USA). Small RNA libraries were constructed according to the manufacturer's instructions using the NEB Next Multiplex Small RNA Library Prep Set (New England Biolabs, USA.). Small RNA sequencing library was sequenced on a Hiseq platform (Illumina). The distribution of vsiRNAs along the viral reference sequence genome (ftp://ftp.ncbi.nlm.nih.gov/ genomes/refseq/viral/) were determined using Bowtie2, and the nucleotide coverage and consensus at each site of the contigs was determined using SAMtools. The results show that the most and total of 2,187,509 reads $(6.08 \%$ in all) in row data were mapped to the complete genome of $\mathrm{NC}_{-}$ 029064 (Cucumis melo endornavirus CL-01) (Sabanadzovic et al. 2016). So, it is speculated that this plant has been infected with the endornavirus. To validate, the dsRNA was extracted from melon tissues using CF-11 cellulose as described by Morris and Dodds (Morris and Dodds 1979), and RT-PCR was performed using primers CmEV-F (5'- GAGGTGAA CACAGCACTTGTTG-3') and CmEV-R (5'- CCAGAGAA TAATGGACAGCCAC-3') to amplify an approximately 710 bp products of the Cucumis melo endornavirus (CmEV) genomic RNA. Nucleotide sequence analysis of the PCR

Fuming Dai

fumingdai@163.com

1 Institute of Eco-Environment and Plant Protection, Shanghai Key

Laboratory of Protection Horticultural Technology, Shanghai

Academy of Agricultural Sciences, Shanghai 201403, China products showed $98.31 \%$ identities with an isolate of $\mathrm{CmEV}$ (SJ1; KX641269.1) infecting C. melo var. makuwa in South Korea (Unpublished). Moreover, by this RT-PCR detection method, we found that the presence of $\mathrm{CmEV}$ on melon with no visible phenotype is a very common phenomenon in Shanghai, China. The symptoms of our siRNA sequenced samples may be caused by physiological disease or other unknown reasons. However, this hypothesis needs further testing based on detailed studies. There have been many reports that endornaviruses are transmitted vertically via seed (eg. Oryza sativa endornavirus and bell pepper endornavirus), and we also have some experimental evidence of the existence of CmEV in melon seedlings of parents, self-crossing 1(S1), S2 and S3. To our knowledge, this is the first report of an endornavirus infecting C. melo in China.

\section{References}

Morris JT, Dodds JA (1979) Isolation and analysis of double-stranded RNA from virus-infected plant and fungal tissue. Phytopathology 69(8):854-858

Sabanadzovic S, Wintermantel WM, Valverde RA, McCreight JD, Aboughanem-Sabanadzovic N (2016) Cucumis melo endornavirus: genome organization, host range and co-divergence with the host. Virus Res 214:49-58

Publisher's note Springer Nature remains neutral with regard to jurisdictional claims in published maps and institutional affiliations. 